\title{
Resultados de la cirugía laparoscópica en el tratamiento electivo de la enfermedad diverticular de colon
}

\author{
Francisco López-Köstner ${ }^{1}$, Alejandro Zárate ${ }^{2}$, G eorge Pinedo², \\ María E. Molina², U do Kronberg ${ }^{1}$, Javiera Pardo². \\ Results of laparoscopic surgery \\ for the treatment of diverticular \\ disease of the colon
}

Background: The laparoscopic approach is an alternative for the elective treatment of diverticular colon disease (DCD). Aim: To analyze the results of patients electively operated for DCD using a laparoscopic technique. Material and Methods: Data of patients with DCD operated using laparoscopy, at the Catholic University of Chile Clinical Hospital were prospectively recorded from January 1999 to August 2006. Indications for surgery were repetitive crises of acute diverticulitis, the persistence of the symptoms or anatomic deformity after the first crisis and complicated diverticulitis (Hinchey 1-2) that responded to the medical treatment. The laparoscopic technique used five ports and the surgical specimen was extracted through a suprapubic approach. Results: One hundred and six patients aged 32 to 82 years ( $49 \%$ females) were operated in the study period. Fifty five percent had a previous abdominal surgery. The mean operative time was 213 minutes (range: 135-360). Four patients were converted to open surgery (3.7\%). One or more early post-operative complications were observed in five patients (4.7\%). The mean time for passing gases and reinitiate liquid diet was 1.7 and 2.4 days respectively. The median post operative stay after surgery was 4 days. There was no operative mortality. Mean follow-up time was 27 months and only one patient $(0.9 \%)$ had a new episode of acute diverticular disease, with a satisfactory response to medical treatment. No patient has developed bowel obstruction. Conclusions: The laparoscopic approach is a safe alternative in the elective surgical treatment of DCD (Rev Méd Chile 2008; 136: 594-9).

(Key words: Diverticulitis; Diverticulum, colon; Laparoscopy)

Recibido el 3 de septiembre, 2007. Aceptado el 24 de enero, 2008.

${ }^{1}$ Unidad de Coloproctología, Clínica Las Condes. Santiago de Chile. ${ }^{2}$ Departamento de Cirugía Digestiva, Pontificia Universidad Católica de Chile. Santiago de Chile.

Correspondencia a: Dr. Francisco López-Köstner. Unidad de Coloproctología, Clínica Las Condes. Lo Fontecilla 441, Las Condes, Santiago. Teléfono: 4888101. Fax: 4888129. E mail: flopez@clinicalascondes.cl 
L a enfermedad diverticular del colon se caracteriza por ser una patología que aumenta su prevalencia en la medida que las personas avanzan en edad. En los países europeos y en Estados Unidos de Norteamérica afecta a sobre $50 \%$ de las personas cuando la población estudiada es mayor de 60 años ${ }^{1,2}$. La forma más frecuente de complicación es la inflamación de los divertículos, siendo el colon sigmoides la ubicación más frecuente de esta complicación lo que puede traducirse en una morbilidad elevada, pudiendo necesitar una resolución quirúrgica de urgencia 0 electiva en casos seleccionados ${ }^{3,4}$.

La sigmoidectomía con anastomosis primaria es la técnica de elección para el tratamiento quirúrgico electivo en estos pacientes ${ }^{5,6}$. Esta operación es técnicamente compleja dado los procesos inflamatorios previos de la zona, sus posibles adherencias y la deformación anatómica del colon sigmoides. Sumado a todo esto, los pacientes sometidos a esta cirugía, en un porcentaje considerable, son mayores de 65 años y presentan variadas comorbilidades, lo cual los expone a diversas complicaciones en el postoperatorio.

Durante años el abordaje tradicional ha sido mediante laparotomía, sin embargo, en la década pasada, mediante el advenimiento del abordaje laparoscópico para patologías intestinales ${ }^{7-9}$, se comenzó a tratar la enfermedad diverticular mediante este último abordaje. Una serie de reportes de estudios comparativos no aleatorios entre el abordaje laparoscópico y mediante laparotomía ${ }^{10-17}$ se han publicado en los últimos diez años, señalando como posibles ventajas de este método una recuperación más rápida del íleo postoperatorio, estadía hospitalaria abreviada, una menor tasa de complicaciones y un mejor resultado estético entre otras variables.

El objetivo de este trabajo, es analizar los resultados de un protocolo prospectivo de tratamiento quirúrgico laparoscópico, en pacientes operados en forma electiva por enfermedad diverticular del colon.

\section{MATERIALES Y MÉTOdOS}

Mediante un protocolo de estudio prospectivo que se inició en 1998, se incluyeron a todos los pacientes operados mediante abordaje laparoscó- pico por enfermedades intestinales, en el Hospital Clínico de la Universidad Católica de Chile ${ }^{18}$. Los datos de los pacientes sometidos a cirugía fueron incluidos prospectiva y consecutivamente en una base de datos computacional. Para este trabajo se seleccionaron los pacientes operados con diagnóstico preoperatorio de enfermedad diverticular, que fuesen operados en forma electiva. Se excluyeron los pacientes con diagnóstico de enfermedad diverticular complicada que debiesen ser operados de urgencia. El periodo de estudio comprende entre enero de 1999 y agosto de 2006. Las indicaciones operatorias fueron diverticulitis recurrente, persistencia de síntomas o deformidad anatómica luego del primer episodio de diverticulitis y el episodio de diverticulitis complicada (Hinchey 1-2) que responde a tratamiento médico. Todas las cirugías consistieron en una resección de colon sigmoides más una extensión variable del colon izquierdo (colectomía segmentaria) con anastomosis primaria mecánica intracorpórea, sin ostomía de protección.

Todos los pacientes fueron operados previa anestesia general, con el paciente en posición Lloyd Davies, con el uso rutinario de pierneras Dan Allen, sonda Foley y una dosis de antibióticos intravenosa en el preoperatorio. Se realizó la insuflación del neumoperitoneo mediante una aguja de Verres por la incisión umbilical. Posteriormente se completó la inserción de 5 trócares. Se usaron 2 trócares de $10 \mathrm{~mm}$ y 3 de $5 \mathrm{~mm}$. La óptica fue de $0^{0}$ y en general, la cámara se ubicó en el trócar umbilical. La disección del retroperitoneo se abordó desde medial hacia lateral, hasta la visualización del uréter izquierdo, para luego seccionar los vasos mesentéricos en su origen. La sección de la pieza operatoria a distal fue a nivel del promontorio sacro y a proximal en una zona del colon donde la palpación mostrara un colon de consistencia y grosor conservado. En otras palabras, no fue el objetivo de la operación la extirpación de todos los divertículos sino la de la zona inflamatoria/fibrosa del colon sigmoides que en algunos casos se extendía a proximal. Se extrajo la pieza operatoria mediante una minilaparotomía horizontal suprapúbica. De rutina se practicó el descenso reglado del ángulo esplénico del colon, salvo excepciones. La anastomosis fue realizada en forma intracorpórea término-terminal bajo la línea del promontorio mediante una 
corchetera mecánica circular no 29 a 33 mm. El uso de drenaje no fue rutinario.

Se registraron y analizaron los datos demográficos, indicación de la cirugía, riesgo anestésico según clasificación de ASA (American Society of Anesthegiologists), variables clínicas prequirúrgicas, intraoperatorias, complicaciones, seguimiento a corto y mediano plazo. Para el seguimiento de los pacientes se usó la escala de Wexner ${ }^{19}$, la cual se realizó mediante encuesta telefónica o entrevista con su cirujano tratante a cada paciente en forma prospectiva, luego los datos fueron adjuntados a la base computacional.

\section{RESULTADOS}

En el periodo descrito se realizaron 306 cirugías laparoscópicas colorrectales en el marco del protocolo previamente descrito, de las cuales 106 (35\%) fueron sigmoidectomías por enfermedad diverticular, quienes constituyen el grupo de estudio. La edad promedio fue de 54 años (i: 3282), 34 pacientes tenían 60 o más años al momento de la cirugía. Correspondieron al sexo femenino 52 pacientes (49\%).

Según la clasificación de ASA fueron I, II y III, un total de 50, 55 y 1 pacientes, respectivamente. La descripción general del grupo se resume en la Tabla 1.

El tiempo operatorio promedio fue de $213 \mathrm{~min}$ (i: $135-360 \mathrm{~min}$ ).

Cuatro pacientes $(3,7 \%)$ fueron convertidos a cirugía abierta, en todos ellos fue por dificultades anatómicas (3 por dificultad en el descenso del ángulo esplénico y uno por un plastrón peridi- verticular), no siendo ninguna conversión derivada de complicaciones intraoperatorias. En el periodo postoperatorio temprano ( $<30$ días), se observaron una o más complicaciones quirúrgicas en 5 pacientes $(4,7 \%)$. Solamente en un paciente se requirió la transfusión de una unidad de glóbulos rojos $(0,9 \%)$. Ningún paciente desarrolló trombosis venosa profunda, ni tromboembolismo pulmonar. No hubo mortalidad operatoria. En un seguimiento promedio de 26,3 meses, cuatro $(3,7 \%)$ pacientes fueron reoperados. Tres de ellos por hernias incisionales (orificio del trocar de $10 \mathrm{~mm}$ e incisión suprapúbica) y uno por estenosis de la anastomosis. Un paciente $(0,9 \%)$ presentó un nuevo episodio de diverticulitis que respondió satisfactoriamente al tratamiento médico con terapia antibiótica y medidas generales. Ningún paciente ha desarrollado un íleo mecánico (Tabla 2).

El tiempo promedio a la expulsión de gases y la realimentación con régimen líquido fue de 1,7 y 2,4 días, respectivamente. La media de estadía postoperatoria fue de 4 días. Sólo 32 (30,1\%) pacientes requirieron de unidades de intermedio quirúrgico.

En el tiempo de seguimiento mencionado, ningún paciente refirió incontinencia a deposiciones líquidas o sólidas.

\section{DISCUSIÓN}

El abordaje clásico hasta hace unos años para pacientes con indicación quirúrgica por enfermedad diverticular de colon, era la sigmoidectomía mediante una laparotomía (vía abierta) ${ }^{5,6}$. A co-

\section{Tabla 1. D escripción general de pacientes sometidos a sigmoidectomía laparoscópica por enfermedad diverticular}

\begin{tabular}{|lr|}
\hline Variable & Valor \\
\hline Total cirugías laparoscópicas & 306 \\
Sigmoidectomías por enfermedad diverticular & $106 \quad(35 \%)$ \\
Edad promedio en años & 54 \\
Sexo femenino & $52(49 \%)$ \\
ASA I & 50 \\
II & 55 \\
III & 1 \\
\hline
\end{tabular}


Tabla 2. C omplicaciones postoperatorias a corto y largo plazo de pacientes sometidos a sigmoidectomía laparoscópica por enfermedad diverticular

\begin{tabular}{|lc|}
\hline Complicaciones & № \\
\hline Precoces (<30 días) & 5 \\
Infección herida operatoria & 3 \\
Sangrado anastomótico & 1 \\
Hematoma suprapúbico & 1 \\
Tardías ( >30 días) & 4 \\
Hernias incisionales & 3 \\
Estenosis anastomosis & 1 \\
\hline
\end{tabular}

mienzos de la década 1990-99, se introdujo la cirugía laparoscópica intestinal para el tratamiento de diversas patologías ${ }^{8,9}$. Una de las patologías que se comenzó a tratar mediante esta vía de abordaje, fue la enfermedad diverticular de colon ${ }^{7}$. Los principios quirúrgicos, en cuanto a los límites proximal y distal de la resección del colon, son los mismos que mediante el abordaje por laparotomía. A la fecha, se han publicado por diversos grupos quirúrgicos estudios comparativos entre el abordaje abierto y el laparoscópico, destacando ventajas en cuanto a la estadía postoperatoria, menor íleo postoperatorio, menor requerimiento de analgesia y el claro mejor resultado cosmético en el grupo laparoscópico ${ }^{10-17}$.

En nuestro grupo, en 1998 se inició progresivamente el desarrollo del abordaje laparoscópico para patologías intestinales, mediante un protocolo de trabajo, el cual incluía la incorporación de todos los pacientes en una base de datos prospectiva $^{18}$. En 2003 publicamos un estudio comparativo para el tratamiento de la enfermedad diverticular, el cual mostró las ventajas descritas en estudios internacionales a favor del grupo laparoscópico ${ }^{15}$.

Las indicaciones quirúrgicas expuestas en este trabajo son las más aceptadas en el ámbito quirúrgico internacional 20,21 , y concordantes con las series laparoscópicas de grandes volúmenes de pacientes $^{22}$. Las complicaciones en esta serie son menos de 10\%, lo cual puede expresar la selección de cirugías menos demandantes técnicamente al comienzo de la serie, para luego ir aumentando progresivamente el grado de complejidad.
La realimentación de los pacientes es uno de los factores para indicar el alta hospitalaria de los pacientes. Usamos como uno de los factores a considerar para la realimentación, el tiempo a la expulsión de gases, evento que ocurrió en general al segundo día postquirúrgico, motivo por el cual, la realimentación con líquidos también se reinició ese día. No observamos mayores complicaciones relacionadas a la realimentación de los pacientes.

En general, al primer día postoperatorio los pacientes deambularon sin grandes dificultades. Consideramos a este hecho, junto con la protección con heparinas de bajo peso molecular, como factores protectores de la trombosis venosa profunda. Esta complicación no la observamos en ningún paciente de la serie, lo cual se compara de forma aceptable para las diversas cifras publicadas que varían entre $0 \%$ y $41 \%$ de presencia de trombosis en extremidades inferiores ${ }^{23-25}$.

Así, una deambulación precoz e ingesta de régimen el segundo día postoperatorio son hechos importantes a considerar al momento de indicar un alta con el paciente movilizándose e ingiriendo dieta liviana al cuarto día sin mayores dificultades. La cantidad de días de estadía postoperatoria de nuestros pacientes se compara favorablemente con las series de 100 o más pacientes operados en el ámbito internacional ${ }^{26-29}$.

En el seguimiento, que en general fue a los dos años de la cirugía, observamos que una nueva crisis clínica de diverticulitis aguda se evidenció solamente en un paciente, el cual se trató médicamente sin dificultades.

Como otra potencial ventaja al realizar esta operación mediante laparoscopia, se encuentra el posible menor desarrollo de adherencias ${ }^{30}$. Si bien en nuestro seguimiento no hemos observado cuadros de íleo mecánico, un seguimiento a mayor plazo confirmará la ventaja previamente enunciada.

$\mathrm{Si}$ bien algunos de los resultados obtenidos mediante el abordaje laparoscópico pueden ser obtenidos con protocolos abreviados de cirugía abierta con incisiones pequeñas ${ }^{31,32}$, en cuanto a una corta estadía hospitalaria y rápida realimentación, la seguridad obtenida al tener una completa visión de la cavidad abdominal, poder descender el ángulo esplénico y mantener los principios quirúrgicos de la cirugía clásica hacen que la vía laparoscópica presente otras ventajas sobre los protocolos de laparotomía pequeñas ${ }^{33,34}$. 
En resumen, la cirugía laparoscópica no solamente es una alternativa segura para el tratamiento electivo de los pacientes con enfermedad diverticular, sino también muestra beneficios evi-

\section{REFERENCIAS}

1. Ferzoco LB, Raptopoulos V, Silen W. Acute diverticulitis. N Engl J Med 1998; 338: 1521-6.

2. Comparato G, Pilotto A, Franze A, Franceschi M, Di Mario F. Diverticular disease in the elderly. Dig Dis 2007; 25: 151-9.

3. MäKelä J, Vuolo S, Kiviniemi H, Laitinen S. Natural history of diverticular disease: when to operate? Dis Colon Rectum 1998; 41: 1523-8.

4. Vignati PV, Welch JP, Cohen JL. Long-term management of diverticulitis in young patients. Dis Colon Rectum 1995; 38: 627-9.

5. ZúÑIGa A, Viñuela E, López F, Rahmer A. Enfermedad diverticular del colon. Resultados del tratamiento quirúrgico electivo. Rev Chil Cir 2001; 53: 342-6.

6. Bannura G, Contreras J, Melo C, Barrera A, Soto $\mathrm{D}$, MANSILA J. [Indications and long term results of elective surgery for sigmoid diverticular disease]. Rev Méd Chile 2005; 133: 1037-42.

7. JaCobs M, Verdeja JC, GoldStein HS. Minimally invasive colon resection (laparoscopic resection). Surg Laparosc Endosc 1991; 1: 144-50.

8. FowLer DL, White SA. Laparoscopy-assisted sigmoid resection. Surg Laparosc Endosc 1991; 1: 183-8.

9. Cooperman AM, Katz V, Zimmon D, Botero G. Laparoscopic colon resection: A case report. J Laparoendosc Surg 1991; 1: 221-4.

10. Liberman MA, Phimps EH, Carroll BJ, Fallas M, RoSENTHAL R. Laparoscopic colectomy vs traditional colectomy for diverticulitis. Outcome and costs. Surg Endosc 1996; 10: 15-8.

11. Stocchi L, Nelson H, Young-Fadok TM, Larson DR, ILSTRUP DM. Safety and advantages of laparoscopic vs open colectomy in the elderly: matchedcontrol study. Dis Colon Rectum 2000; 43: 326-32.

12. Faynsod M, Stamos MJ, Arnell T, Borden C, Udani M, VARGAS H. A case-control study of laparoscopic versus open sigmoid colectomy for diverticulitis. Am Surg 2000; 66: 841-3.

13. Dwivedi A, Chahin F, Agrawal S, Chau WY, Tootla A, Toотla F ET AL. Laparoscopic colectomy vs. dentes. Se sugiere este abordaje como la vía de elección, para el tratamiento electivo de los pacientes con indicación quirúrgica por enfermedad diverticular de colon.

open colectomy for sigmoid diverticular disease. Dis Colon Rectum 2002; 45: 1309-15.

14. Senagore AJ, Duepree HJ, Delaney CP, Dissanaike S, BRADY KM, FAZIo VW. Cost structure of laparoscopic and open sigmoid colectomy for diverticular disease: similarities and differences. Dis Colon Rectum 2002; 45: 485-90.

15. López F, Soto G, Tapia G, Schnettler K, Zárate A, Avendano R ET AL. [Elective laparoscopic surgery in diverticular disease. A comparative study with conventional operative surgery]. Rev Méd Chile 2003; 131: 719-26.

16. Lawrence DM, Pasquale MD, Wasser TE. Laparoscopic versus open sigmoid colectomy for diverticulitis. Am Surg 2003; 69: 499-503.

17. González R, Smith CD, Mattar SG, Venkatesh KR, Mason E, Duncan T ET al. Laparoscopic vs open resection for the treatment of diverticular disease. Surg Endosc 2004; 18: 276-80.

18. López-Köstner F, Soto G, Zárate A, Pinedo G, Pérez G, Avendaño R et al. Protocolo de cirugía laparoscópica intestinal. Rev Chil Cir 2003; 55: 225-31.

19. Jorge JM, WeXNER SD. Etiology and management of fecal incontinence. Dis Colon Rectum 1993; 36: 77-97.

20. KöHler L, SauerLand S, Neugebauer E. Diagnosis and treatment of diverticular disease. Results of a consensus development conference. The Scientific Committee of the European Association for Endoscopic Surgery. Surg Endosc 1999; 13: 430-6.

21. Senagore AJ. Laparoscopic sigmoid colectomy for diverticular disease. Surg Clin NAm 2005; 85: 19-24.

22. KoCKerLing F, SchNeider C, REYMond MA, Scheidbach H, Scheuerlein H, KonRadt J et al. Laparoscopic resection of sigmoid diverticulitis. Results of a multicenter study. Laparoscopic Colorectal Surgery Study Group. Surg Endosc 1999; 13: 567-71.

23. Schenk W, Bohm B, Junghans T, Hofmann H, MuLeR JM. Intermittent sequential compression of the lower limbs prevents venous stasis in laparoscopic and conventional colorectal surgery. Dis Colon Rectum 1997; 40: 1056-62.

24. McLeod RS, Geerts WH, SNiderman KW, Greenwood C, Gregoire RC, Taylor BM et al; Canadian Colo- 
rectal Surgery DVT ProphylaXis Trial Investigators. Subcutaneous heparin versus low-molecularweight heparin as thromboprophylaxis in patients undergoing colorectal surgery: results of the canadian colorectal DVT prophylaxis trial: a randomized, double-blind trial. Ann Surg 2001; 233: 438-44.

25. Lee FY, Chu W, Chan R, Leung YF, Liu KH, Ng SM ET AL. Incidence of deep vein thrombosis after colorectal surgery in a Chinese population. ANZ J Surg 2001; 71: 637-40.

26. Wilhelm T, Refeidi A, Palma P, Neufang T, Post S. Hand-assisted laparoscopic sigmoid resection for diverticular disease: 100 consecutive cases. Surg Endosc 2006; 20: 477-81.

27. Trebuchet G, LechauX D, Lecalve J. Laparoscopic left colon resection for diverticular disease. Results from 170 consecutive cases. Surg Endosc 2002; 16: 18-21.

28. Blake MF, Dwivedi A, Tootia A, Tootla F, Silva YJ. Laparoscopic sigmoid colectomy for chronic diverticular disease. JSLS 2005; 9: 382-5.
29. Bouilot JL, Berthou JC, Champault G, Meyer C, Arnaud JP, Samama G et al. Elective laparoscopic colonic resection for diverticular disease: results of a multicenter study in 179 patients. Surg Endosc 2002; 16: 1320-3.

30. Gutt CN, Oniu T, Schemmer P, Mehrabi A, Buchler MW. Fewer adhesions induced by laparoscopic surgery? Surg Endosc 2004; 18: 898-906.

31. Proske JM, Raue W, Neudecker J, Muler JM, SchwENK W. Fast track rehabilitation in colonic surgery: results of a prospective trial. Ann Chir 2005; 130: 152-6.

32. Basse L, Thorbol Je, Lossl K, Kehlet H. Colonic surgery with accelerated rehabilitation or conventional care. Dis Colon Rectum 2004; 47: 271-7.

33. BenN PL, WolfF BG, ILSTRUP DM. Level of anastomosis and recurrent colonic diverticulitis. Am J Surg 1986; 151: 269-71.

34. Bergamaschi R, Arnaud JP. Anastomosis level and specimen length in surgery for uncomplicated diverticulitis of the sigmoid. Surg Endosc 1998; 12: 1149-51. 